\title{
Outage Performance on Threshold AF and DF Relaying Schemes in Simultaneous Wireless Information and Power Transfer Systems
}

\author{
Gaofeng Pan $^{1}$, Chaoqing Tang ${ }^{2}$, Zhetao $\mathrm{Li}^{3}$ \\ 1. The School of Electronic and Information Engineering, Southwest University, \\ Chongqing, 400715, China \\ 2. School of Electrical \& Electronic Engineering, Newcastle University, Newcastle upon \\ Tyne, NE1 YRU, UK. \\ 3. College of Information Engineering, Xiangtan University, Xiangtan 411105, China.
}

\section{Abstract}

In the work, a dual-hop cooperative simultaneous wireless information and power transfer (SWIPT) system, in where there are a Source-Destination $(S-$ $D)$ pair and a relay node $(R)$, is considered. $R$ adopts threshold amplify-andforward (AF)/decode-and-forward (DF) relaying schemes to decide whether to aid $S-D$ pair's information transmission not. The effect of SWIPT on the outage performance has been investigated and the closed-form analytical expressions for outage probability have been derived under threshold AF and DF schemes, respectively, which are verified by Monte-Carlo simulations. Keywords:

Simultaneous wireless information and power transfer; amplify-and-forward; decode-and-forward; outage probability

Preprint submitted to AEU - International Journal of Electronics and CommunicationsMarch 15, 2016

(C) 2016. This manuscript version is made available under the Elsevier user license http://www.elsevier.com/open-access/userlicense/1.0/ 


\section{Introduction}

Nowadays, energy budget is a crucial limitation factor on mobile terminals' lifetime and performance, which are usually equipped with rechargeable batteries. As a promising solution for this problem, simultaneous wireless information and power transfer (SWIPT) technology was proposed for future wireless communications [1]-[2]. Under SWIPT transmission scheme, the radio-frequency $(\mathrm{RF})$ signals radiated by the transmitters, which are used for information delivery, can also be regarded as a viable source of energy. At the receiver, the received $\mathrm{RF}$ signal can be split into two portions: one for information decoding and the other for energy harvesting, by using power splitting method [3]-[5].

Plenty of attentions have been paid on power control, resource scheduling and transmission schemes design in SWIPT systems [6]-[16]. [6] investigated multiple-input-multiple-output (MIMO) communications under energy harvesting (EH) constraints with partial channel state information (CSI). The authors of [7] proposed an optimal and the low complexity suboptimal energy efficient power allocation algorithms for MIMO SWIPT systems with covariance CSI feedback. In [8], the authors proposed a robust beamforming design for the multi-antenna SWIPT systems to maximize the worst-case harvested energy for the energy-harvesting receiver (ER), as well as to guarantee that the rate at the IR is above a threshold. In a MIMO SWIPT system, the authors of [9] derived closed-form expressions for the energy shortage and data outage probability under the three cases for the CSI knowledge at the transmitter: no CSI, and imperfect CSI in case of time-division duplexing and frequency-division duplexing communications. In a power splitting (PS)- 
based multiple-input single-output (MISO) interference channel for SWIPT, the total transmission power was minimized by joint beamforming and PS under both the SINR and EH constraints in the presence of local CSI [10]. The authors of [11] investigated the joint multicast transmit beamforming and receive power splitting problem for MISO SWIPT systems and minimized the transmit power subject to signal-to-noise ratio (SNR) and EH constraints at each receiver, in both scenarios of perfect and imperfect CSI at the transmitter. In [12], two robust joint beamforming and power splitting designs were developed for a downlink MISO SWIPT system to minimize the transmission power under both the SINR and EH constraints per user. In a MIMO SWIPT system, a robust secure transmission scheme was proposed and investigated to maximize the worst-case secrecy rate under transmitting power constraint and EH constraint while considering channel uncertainties [13]. In [14], a robust artificial noise-aided secure transmission design was proposed for MIMO SWIPT systems, while considering the worst-case channel uncertainties model. In a MISO SWIPT system, the harvested energy by the ERs was maximized while maintaining the SINR threshold at the IR [16].

Inspired from the above observations, in this work, a cooperation group including a Source-Destination $(S-D)$ pair, and a relay node, $R$, is considered. Threshold amplify-and-forward (AF) and decode-and-forward (DF) relaying schemes have been considered at $R$ to process and forward the received signals to $D$. The effect of SWIPT on the outage performance has been studied under both threshold DF/AF relaying schemes, respectively, by setting up the analytical models. The closed-form analytical expressions 
have been derived for threshold $\mathrm{AF} / \mathrm{DF}$ relaying schemes.

However, most of the existed works related to SWIPT systems [3]-[16] are limited to single-hop scenarios and the effect on SWIPT technology on the performance of cooperative systems has not been investigated yet.

The rest of the paper is structured as follows. In Section 2, the system model is introduced. The outage performance is studied for threshold AF and DF schemes, respectively, as well as deriving the closed-form analytical expressions for the outage probability of the considered system, in Section 3. The proposed analytical models are evaluated by Monte-Carlo simulations in Section 4. Finally, the paper is concluded in Section 5.

\section{System model}

In this section, the simplest cooperation group including a Source-Destination $(S-D)$ pair, and a relay node, $R$, is considered. All links experience independent and identically Rayleigh fading. It is assumed that each terminal has a single antenna and operates in a half-duplex mode.

$R$ adopts power splitting method to coordinate the processes of informa-

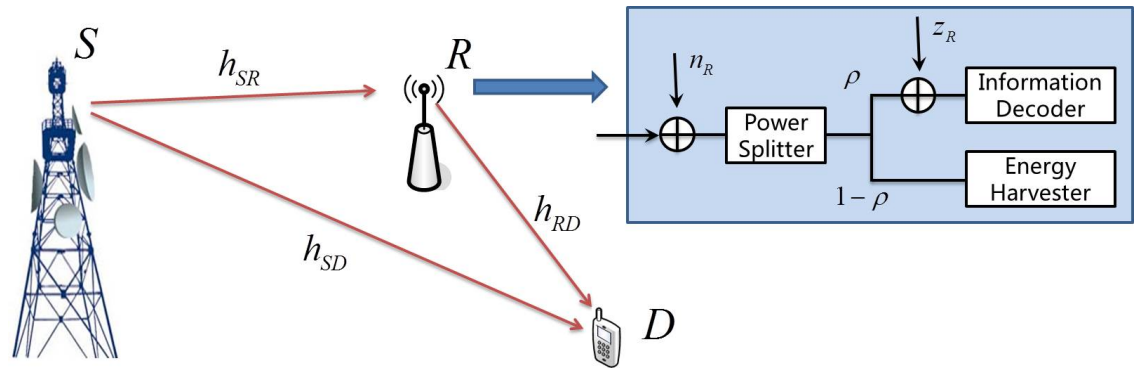

Figure 1: Diagram of cooperative SWIPT system model

tion decoding and energy harvesting from the received signal [17]. Specifi- 
cally, the received signal at $R$ is split to the information decoder (ID) and the energy harvester $(\mathrm{EH})$ by a power splitter, which divides an $(\rho)$ portion of the signal power to the ID, and the remaining portion $(0 \leq \rho \leq 1)$ of power to the EH.

The information transmission process can be divided into two phases: 1) $S$ broadcasts the information to $R$ and $D ; 2) R$ adopts its instantaneous SNR of the received signal at its IR as an indication of the reliability of the relay detection. If the SNR is larger than a predefined threshold, the probability of an error at the relay is small under DF scheme or the quality of the received signal is good enough under AF scheme. Hence $R$ forwards the signal after recoding under DF scheme or amplifying under AF. Otherwise, $R$ remains silent.

In Phase 1, the downlink received signals at the IR of $R$ and $D$ are given by

$$
\begin{array}{cc}
y_{R}=\sqrt{\rho}\left(\sqrt{P_{S}} h_{S R} s+n_{R}\right)+z_{R} & (1-a) \\
y_{D 1}=\sqrt{P_{S}} h_{S D} s+n_{D}, & (1-b)
\end{array}
$$

respectively, where $P_{i}(i \in\{S, R\})$ is the transmitting power at node $i, s$ denotes the transmitted symbols from BS, $h_{i j}(i, j \in\{S, D, R\})$ is the link channel gain between node $i$ and $j, n_{i}(i \in\{D, R\})$ denotes the independent complex Gaussian noise at the IR of $R$ and $D$. In this work, to simplify the analysis, we assume that and are with zero means and a same variances, $N_{0}$. $z_{R}$ is the signal processing noise by the ID at $R$, which can also be modeled as additional white Gaussian noise with zero means and variances $\sigma^{2}$.

Therefore, the SNR of the received signal at IR of $R$ and $D$ can be written 
as

$$
\begin{gathered}
\gamma_{R}=\frac{\rho P_{S}\left|h_{S R}\right|^{2}}{\rho N_{0}+\sigma^{2}} \\
\gamma_{D 1}=\frac{P_{S}\left|h_{S D}\right|^{2}}{N_{0}} .
\end{gathered}
$$

The probability density function (PDF) of $\left|h_{S R}\right|^{2}$ and $\left|h_{S D}\right|^{2}$ can be given as

$$
\begin{aligned}
& f_{\left|h_{S R}\right|^{2}}(x)=\frac{1}{h_{1}} \exp \left(-\frac{x}{h_{1}}\right) \\
& f_{\left|h_{S D}\right|^{2}}(x)=\frac{1}{h_{2}} \exp \left(-\frac{x}{h_{2}}\right),
\end{aligned}
$$

respectively, where $h_{1}$ and $h_{2}$ are the expectation of channel power gain $\left|h_{S R}\right|^{2}$ and $\left|h_{S D}\right|^{2}$, respectively.

Further, we can obtain $\gamma_{R} \sim \operatorname{Exp}\left(\lambda_{S R}\right)$ and $\gamma_{D 1} \sim \operatorname{Exp}\left(\lambda_{S D}\right)$, where $\lambda_{S R}=$ $\frac{\rho N_{0}+\sigma^{2}}{h_{1} \rho P_{S}}$ and $\lambda_{S D}=\frac{N_{0}}{h_{2} P_{S}}$.

\section{Outage analysis}

\subsection{DF scheme}

In the second phase, if $R$ decides to forward the detected symbol, $s_{R}$, to $D$ after regenerating, the received signal at $D$ is

$$
y_{D 2}=\sqrt{P_{R}} h_{R D} s_{R}+n_{D}
$$

Therefore, the SNR of the received signal at $D$ can be written as

$$
\gamma_{D 2}=\frac{P_{R}\left|h_{R D}\right|^{2}}{N_{0}} .
$$

The PDF of $\left|h_{R D}\right|^{2}$ can be written as $f_{\left|h_{R D}\right|^{2}}(x)=\frac{1}{h_{3}} \exp \left(-\frac{x}{h_{3}}\right)$, where $h_{3}$ is the expectation of channel power gain $\left|h_{R D}\right|^{2}$. Then, we can also obtain 
$\gamma_{D 2} \sim \operatorname{Exp}\left(\lambda_{R D}\right)$, where $\lambda_{R D}=\frac{N_{0}}{h_{3} P_{R}}$.

In this work, we assume that $D$ adopts $\mathrm{SC}$ scheme to combine $y_{D 1}$ and $y_{D 2}$ as the final received signal. Then, we have $\gamma_{D}=\left\{\begin{array}{cc}\gamma_{D 1} & \text { if } \gamma_{S R} \leq \gamma_{\text {th }} \\ \max \left\{\gamma_{D 1}, \gamma_{D 2}\right\} & \text { else }\end{array}\right.$.

The outage probability for the considered system using threshold can be expressed as

$$
\begin{gathered}
\operatorname{Pr}_{\text {out }}\left(\gamma_{\text {th }}\right)=\operatorname{Pr}\left\{\gamma_{S R}>\gamma_{\text {th }}\right\} \operatorname{Pr}\left\{\gamma_{D} \leq \gamma_{\text {th }} \mid \gamma_{S R}>\gamma_{\text {th }}\right\} \\
+\operatorname{Pr}\left\{\gamma_{S R} \leq \gamma_{\text {th }}\right\} \operatorname{Pr}\left\{\gamma_{D 1} \leq \gamma_{\text {th }}\right\}
\end{gathered}
$$

where $\gamma_{D}$ denotes the SNR of the output signal at the combiner of $D$, which can be written as $\gamma_{D}=\max \left\{\gamma_{D 1}, \gamma_{D 2}\right\}$.

In the following, we derive the terms in (6), respectively.

The probability that the SNR of the $S-R$ link $\gamma_{S R}$ is below the threshold can be given as

$$
\operatorname{Pr}\left\{\gamma_{S R} \leq \gamma_{\mathrm{th}}\right\}=\int_{0}^{\gamma_{\mathrm{th}}} \lambda_{S R} \exp \left(-\lambda_{S R} x\right) d x=1-\exp \left(-\lambda_{S R} \gamma_{\mathrm{th}}\right) .
$$

Similarly, we can obtain the probability that the SNR of the $S-D$ link is below the threshold as

$$
\operatorname{Pr}\left\{\gamma_{S D} \leq \gamma_{\text {th }}\right\}=\int_{0}^{\gamma_{\text {th }}} \lambda_{S D} \exp \left(-\lambda_{S D} x\right) d x=1-\exp \left(-\lambda_{S D} \gamma_{\text {th }}\right) .
$$

The probability that the SNR of the combined signal at $D, \gamma_{D}$, is below the threshold can be given as

$$
\begin{gathered}
\operatorname{Pr}\left\{\gamma_{D} \leq \gamma_{\text {th }} \mid \gamma_{S R}>\gamma_{\text {th }}\right\}=\operatorname{Pr}\left\{\gamma_{D} \leq \gamma_{\text {th }}\right\} \\
=\operatorname{Pr}\left\{\max \left\{\gamma_{D 1}, \gamma_{D 2}\right\} \leq \gamma_{\text {th }}\right\}
\end{gathered}
$$




$$
=\left[1-\exp \left(-\lambda_{S D} \gamma_{\mathrm{th}}\right)\right]\left[1-\exp \left(-\lambda_{R D} \gamma_{\mathrm{th}}\right)\right]
$$

Therefore, SOP can be obtained by substituting (7), (8) and (9) into (6) as

$$
\begin{aligned}
\operatorname{Pr}_{\text {out }}\left(\gamma_{\mathrm{th}}\right)= & \exp \left(-\lambda_{S R} \gamma_{\mathrm{th}}\right) \cdot\left[1-\exp \left(-\lambda_{S D} \gamma_{\mathrm{th}}\right)\right]\left[1-\exp \left(-\lambda_{R D} \gamma_{\mathrm{th}}\right)\right] \\
& +\left[1-\exp \left(-\lambda_{S R} \gamma_{\mathrm{th}}\right)\right] \cdot\left[1-\exp \left(-\lambda_{S D} \gamma_{\mathrm{th}}\right)\right]
\end{aligned}
$$

\subsection{AF scheme}

In the second phase, if $R$ decides to forward the detected symbol, $s_{R}$, to $D$ after amplifying, the received signal at $D$ can be written as

$$
\begin{gathered}
y_{D 2}=G h_{R D} y_{R}+n_{D} \\
=G h_{R D}\left[\sqrt{\rho}\left(\sqrt{P_{S}} h_{S R} s+n_{R}\right)+z_{R}\right]+n_{D} \\
=\frac{\sqrt{P_{S}}}{\sqrt{P_{S}\left|h_{S R}\right|^{2}+N_{0}}} h_{R D}\left[\sqrt{\rho}\left(\sqrt{P_{S}} h_{S R} s+n_{R}\right)+z_{R}\right]+n_{D} \\
=\frac{P_{S} \sqrt{\rho}}{\sqrt{P_{S}\left|h_{S R}\right|^{2}+N_{0}}} h_{R D} h_{S R} s+\frac{\sqrt{P_{S}} h_{R D}}{\sqrt{P_{S}\left|h_{S R}\right|^{2}+N_{0}}}\left(\sqrt{\rho} n_{R}+z_{R}\right)+n_{D},
\end{gathered}
$$

where $G=\frac{\sqrt{P_{S}}}{\sqrt{P_{S}\left|h_{S R}\right|^{2}+N_{0}}}$ is the amplifying factor at $R$.

As the noise terms $n_{R}, n_{D}$ and $z_{R}$ are independent, $y_{D 2}$ can be rewritten as

$$
y_{D 2}=\frac{P_{S} \sqrt{\rho}}{\sqrt{P_{S}\left|h_{S R}\right|^{2}+N_{0}}} h_{R D} h_{S R} s+n_{D}^{\prime},
$$

where $n_{D}^{\prime}=\frac{\sqrt{P_{S}} h_{R D}}{\sqrt{P_{S}\left|h_{S R}\right|^{2}+N_{0}}}\left(\sqrt{\rho} n_{R}+z_{R}\right)+n_{D}$ is the equivalent noise, which is a zero-mean, complex Gaussian random variable with variance

$$
N_{0}{ }^{\prime}=\frac{P_{S}\left|h_{R D}\right|^{2}}{P_{S}\left|h_{S R}\right|^{2}+N_{0}}\left(\rho N_{0}+\sigma^{2}\right)+N_{0} .
$$


Therefore, the SNR of the received signal at $D$ can be written as

$$
\begin{gathered}
\gamma_{D 2}=\frac{P_{S}^{2} \rho\left|h_{S R}\right|^{2}\left|h_{R D}\right|^{2}}{N_{0}{ }^{\prime}\left(P_{S}\left|h_{S R}\right|^{2}+N_{0}\right)} \\
=\frac{\frac{P_{S}^{2} \rho\left|h_{S R}\right|^{2}\left|h_{R D}\right|^{2}}{P_{S}\left|h_{R}\right|^{2}+N_{0}}}{\frac{P_{S}\left|h_{R D}\right|^{2}}{P_{S}\left|h_{S R}\right|^{2}+N_{0}}\left(\rho N_{0}+\sigma^{2}\right)+N_{0}} \\
=\frac{\rho \frac{P_{S}^{2}}{N_{0}^{2}}\left|h_{S R}\right|^{2}\left|h_{R D}\right|^{2}}{\frac{P_{S}\left(\rho N_{0}+\sigma^{2}\right)}{N_{0}{ }^{2}}\left|h_{R D}\right|^{2}+\frac{P_{S}}{N_{0}}\left|h_{S R}\right|^{2}+1} .
\end{gathered}
$$

At high SNR, the constant 1 in the denominator in (14) can be omitted and the effective SNR of the received signal at $D$ can be approximated as

$$
\begin{aligned}
\gamma_{D 2} \approx & \frac{\rho \frac{P_{S}{ }^{2}}{N_{0}^{2}}\left|h_{S R}\right|^{2}\left|h_{R D}\right|^{2}}{\frac{P_{S}\left(\rho N_{0}+\sigma^{2}\right)}{N_{0}{ }^{2}}\left|h_{R D}\right|^{2}+\frac{P_{S}}{N_{0}}\left|h_{S R}\right|^{2}} \\
= & \frac{\rho \frac{P_{S}}{N_{0}}}{\frac{\left(\rho N_{0}+\sigma^{2}\right)}{N_{0}} \frac{1}{\left|h_{S R}\right|^{2}}+\frac{1}{\left|h_{R D}\right|^{2}}} \\
& =\frac{1}{\frac{a}{b} \frac{1}{\left|h_{S R}\right|^{2}}+\frac{1}{b} \frac{1}{\left|h_{R D}\right|^{2}}},
\end{aligned}
$$

where $a=\frac{\left(\rho N_{0}+\sigma^{2}\right)}{N_{0}}$ and $b=\rho \frac{P_{S}}{N_{0}}$.

As $\left|h_{S R}\right|^{2}$ and $\left|h_{S D}\right|^{2}$ are exponentially distributed with parameters $h_{1}{ }^{-1}$ and $h_{3}{ }^{-1}$, respectively, we can obtain the PDF of $\frac{a}{b} \frac{1}{\left|h_{S R}\right|^{2}}$ and $\frac{1}{b} \frac{1}{\left|h_{R D}\right|^{2}}$ as

$$
\begin{array}{ll}
f_{1}(x)=\frac{a}{b h_{1} x^{2}} \exp \left(-\frac{a}{b h_{1} x}\right) & (16-a) \\
f_{2}(x)=\frac{1}{b h_{3} x^{2}} \exp \left(-\frac{1}{b h_{3} x}\right), & (16-b)
\end{array}
$$

respectively.

Using Eq. (3.471.9) in [18] and the symmetry property of the modified 
Bessel function (as shown by Eq. (8.486.16) in [18]), the moment generating function (MGF) of $Y_{1}=\frac{a}{b\left|h_{S R}\right|^{2}}$ and $Y_{2}=\frac{1}{b\left|h_{R D}\right|^{2}}$ can be given as

$$
\begin{aligned}
& M_{Y_{1}}(s)=2 \sqrt{\frac{a s}{b h_{1}}} K_{1}\left(2 \sqrt{\frac{a s}{b h_{1}}}\right) \\
& M_{Y_{2}}(s)=2 \sqrt{\frac{s}{b h_{3}}} K_{1}\left(2 \sqrt{\frac{s}{b h_{3}}}\right),
\end{aligned}
$$

where $K_{1}(\cdot)$ is the first order modified Bessel function of the second kind defined in [19].

Then, the MGF of $Y=Y_{1}+Y_{2}=\frac{a}{b} \frac{1}{\left|h_{S R}\right|^{2}}+\frac{1}{b} \frac{1}{\left|h_{R D}\right|^{2}}$ can be given as

$$
M_{Y}(s)=M_{Y_{1}}(s) M_{Y_{2}}(s)=4 s \sqrt{\frac{a}{b^{2} h_{1} h_{3}}} K_{1}\left(2 \sqrt{\frac{a s}{b h_{1}}}\right) K_{1}\left(2 \sqrt{\frac{s}{b h_{3}}}\right) .
$$

The CDF of $\gamma_{D 2}, P_{\gamma_{D 2}}(z)$ can be given as

$$
\begin{aligned}
& P_{\gamma_{D 2}}(x)=\operatorname{Pr}\left\{\gamma_{D 2}<x\right\} \\
& =\operatorname{Pr}\left\{\frac{1}{\gamma_{D 2}}>\frac{1}{x}\right\} \\
& =\operatorname{Pr}\left\{Y>\frac{1}{x}\right\} \\
& =1-\operatorname{Pr}\left\{Y<\frac{1}{x}\right\} \\
& =1-P_{Y}\left(\frac{1}{x}\right),
\end{aligned}
$$

where $P_{Y}(\cdot)$ is the CDF of $Y$.

Using the differentiation property of Laplace transform, $P_{Y}(\cdot)$ can be obtained as

$$
P_{Y}(y)=\mathscr{L}^{-1}\left(\frac{M_{Y}(s)}{s}\right)
$$


where $\mathscr{L}^{-1}(\cdot)$ denotes the inverse Laplace transform.

Substituting (18) in (19) and (20) and using Eq. (3.16.13.4) in [20], we have

$$
\begin{aligned}
& P_{\gamma_{D 2}}(x) \approx 1-\left.\mathscr{L}^{-1}\left(4 \sqrt{\frac{a}{b^{2} h_{1} h_{3}}} K_{1}\left(2 \sqrt{\frac{a s}{b h_{1}}}\right) K_{1}\left(2 \sqrt{\frac{s}{b h_{3}}}\right)\right)\right|_{y=\frac{1}{x}} \\
& =1-2 \sqrt{\frac{a}{b^{2} h_{1} h_{3}}} \cdot x \exp \left(-\left(\frac{a}{h_{1}}+\frac{1}{h_{3}}\right) \frac{x}{b}\right) K_{1}\left(2 \sqrt{\frac{a}{h_{1} h_{3}}} \cdot \frac{x}{b}\right) .
\end{aligned}
$$

Therefore, under AF scheme the probability that the SNR of the combined signal at $D, \gamma_{D}$, is below the threshold can be given as

$$
\begin{gathered}
\operatorname{Pr}\left\{\gamma_{D} \leq \gamma_{\mathrm{th}} \mid \gamma_{r}>\gamma_{\mathrm{th}}\right\}=\operatorname{Pr}\left\{\gamma_{D} \leq \gamma_{\mathrm{th}}\right\} \\
=\operatorname{Pr}\left\{\max \left\{\gamma_{D 1}, \gamma_{D 2}\right\} \leq \gamma_{\mathrm{th}}\right\} \\
=\operatorname{Pr}\left\{\gamma_{D 1} \leq \gamma_{\mathrm{th}}\right\} \operatorname{Pr}\left\{\gamma_{D 2} \leq \gamma_{\mathrm{th}}\right\} \\
\approx\left[1-\exp \left(-\lambda_{S D} \gamma_{\mathrm{th}}\right)\right] \\
\cdot\left[1-2 \sqrt{\frac{a}{b^{2} h_{1} h_{3}}} \cdot \gamma_{\mathrm{th}} \exp \left(-\left(\frac{a}{h_{1}}+\frac{1}{h_{3}}\right) \frac{\gamma_{\mathrm{th}}}{b}\right) K_{1}\left(2 \sqrt{\frac{a}{h_{1} h_{3}}} \cdot \frac{\gamma_{\mathrm{th}}}{b}\right)\right]
\end{gathered}
$$

Thus, SOP can be obtained by substituting (7), (8) and (22) into (6) as

$$
\begin{gathered}
\operatorname{Pr}_{\text {out }}\left(\gamma_{\mathrm{th}}\right)=\exp \left(-\lambda_{S R} \gamma_{\mathrm{th}}\right) \cdot\left[1-\exp \left(-\lambda_{S D} \gamma_{\mathrm{th}}\right)\right] \\
\cdot\left[1-2 \sqrt{\frac{a}{b^{2} h_{1} h_{3}}} \cdot \gamma_{\mathrm{th}} \exp \left(-\left(\frac{a}{h_{1}}+\frac{1}{h_{3}}\right) \frac{\gamma_{\mathrm{th}}}{b}\right) K_{1}\left(2 \sqrt{\frac{a}{h_{1} h_{3}}} \cdot \frac{\gamma_{\mathrm{th}}}{b}\right)\right] \\
+\left[1-\exp \left(-\lambda_{S R} \gamma_{\mathrm{th}}\right)\right] \cdot\left[1-\exp \left(-\lambda_{S D} \gamma_{\mathrm{th}}\right)\right]
\end{gathered}
$$




\section{Numerical and simulation results}

To confirm our proposed model, in this section, we compare simulation and analysis results for SOP under both AF and DF schemes. Unless otherwise explicitly specified, the main parameters adopted in this work are set as $P_{s}=P_{R}=1, N_{0}=0.01, \gamma_{\mathrm{th}}=0 \mathrm{~dB}, \rho=0.5, h_{1}=h_{2}=h_{3}=1$.

In Fig. 2, SOP curves for $h_{2}=h_{3}=1$, and $\rho=0.1,0.3,0.5,0.7$ and 0.9 under AF and DF schemes are presented. Clearly, the SOP for a lower $\rho$ outperforms the one for a higher $\rho$. This is because that a lower $\rho$ means a lower portion of the received signal power is split to the ID at the relay, resulting in a higher received SNR at the relay, which leads to a higher capacity. Moreover, SOP gets worse when $h_{3} / h_{1}$ increases because increasing $h_{3} / h_{1}$ means $S-R$ link gets worse, resulting in an increasing outage probability over $S-R$ link.

In Fig. 3, SOP curves for $h_{1}=h_{3}=1$, and $\gamma_{\text {th }}=0,1,2$ and 3 under AF and DF schemes are depicted. It is obvious that the SOP for a lower $\gamma_{\text {th }}$ outperforms the one for a higher $\gamma_{\mathrm{th}}$. Because that a lower $\gamma_{\mathrm{th}}$ means a lower probability that the SNR at each receiver falls below the threshold. Further, SOP gets worse when $h_{1} / h_{2}$ increases because increasing $h_{1} / h_{2}$ means $S-D$ link gets worse, resulting in a lower diversity gain at $D$.

In Fig. 4, SOP curves for $h_{1}=h_{2}$, and $h_{1}=1,2,3$ and 4 under $\mathrm{AF}$ and DF schemes are depicted. One can see that the SOP for a lower $h_{1}$ outperforms the one for a higher $h_{1}$. Because that a lower $h_{1}$ means a lower probability that the SNR at each receiver falls below the threshold. It can also be observed that SOP gets worse when $h_{1} / h_{3}$ increases because 


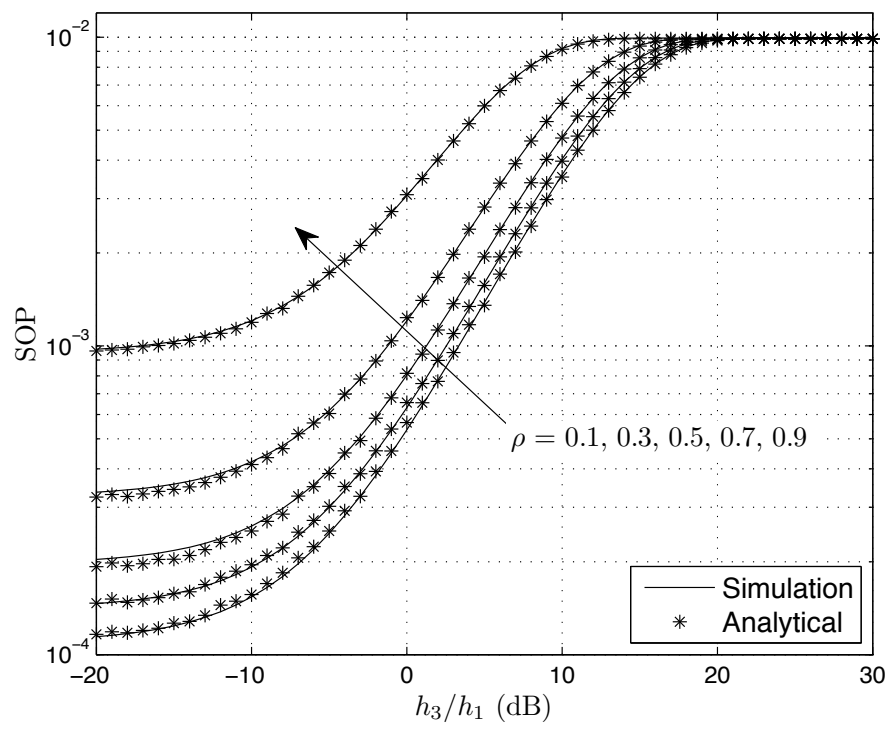

(a) $\mathrm{AF}$

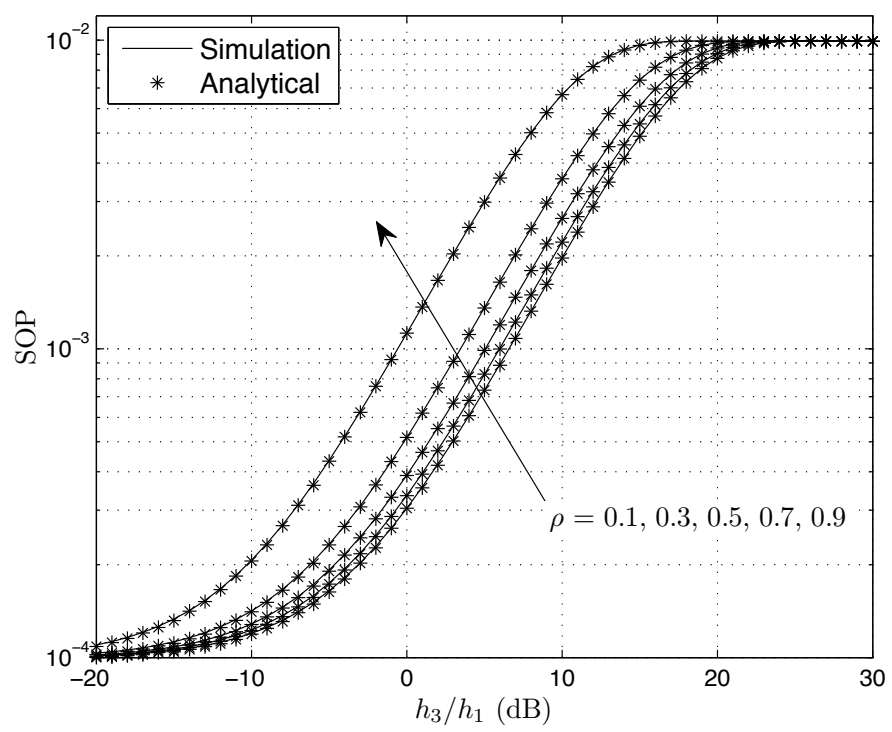

(b) $\mathrm{DF}$

Figure 2: SOP for various $\rho$ 


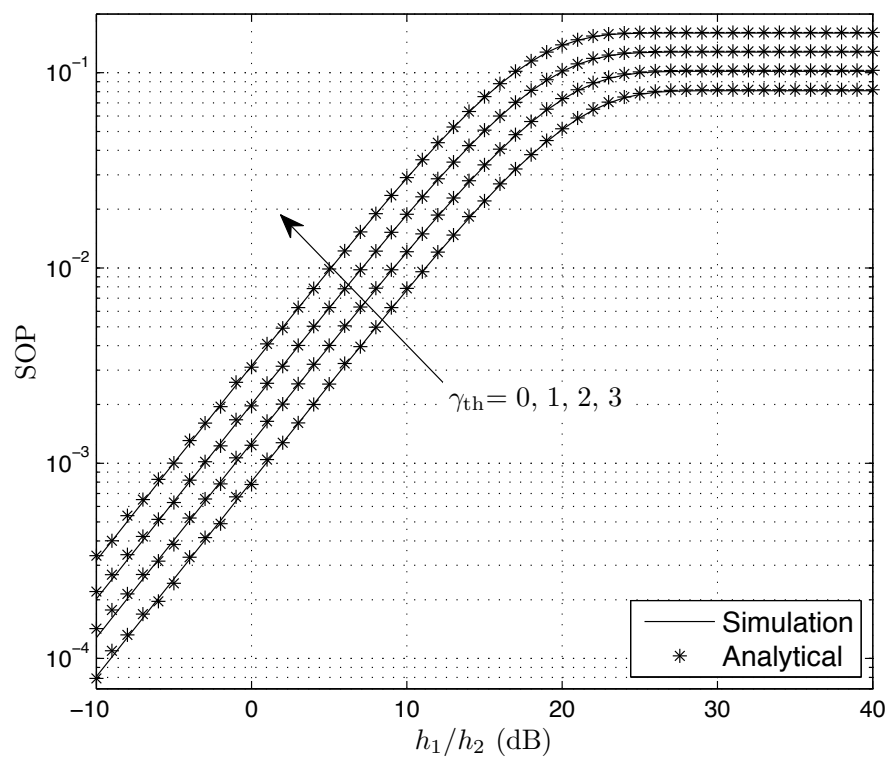

(a) $\mathrm{AF}$

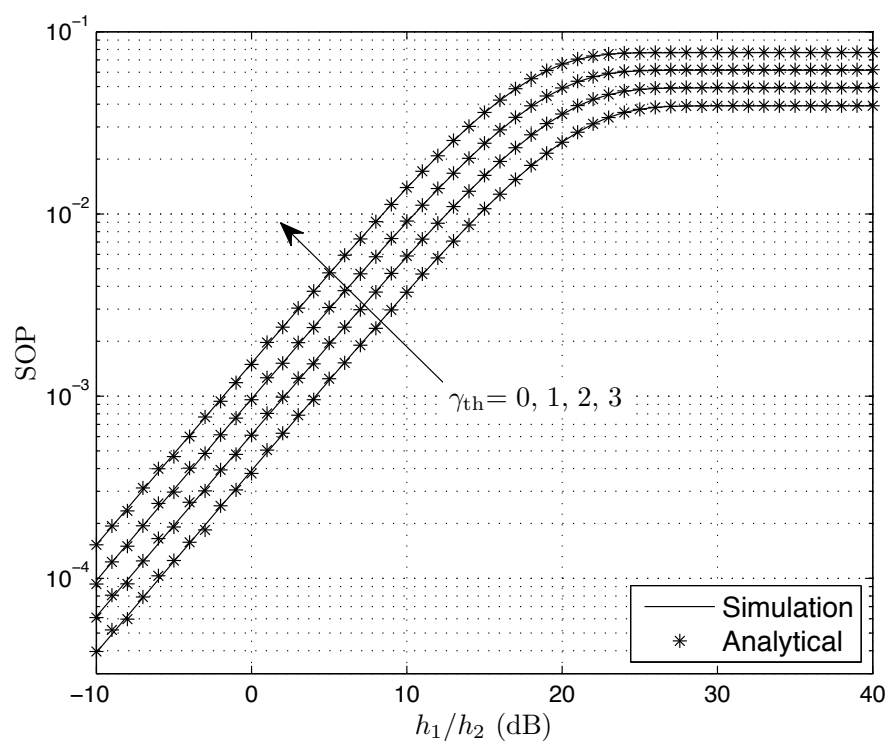

(b) $\mathrm{DF}$

Figure 3: SOP for various $\gamma_{\text {th }}$ 


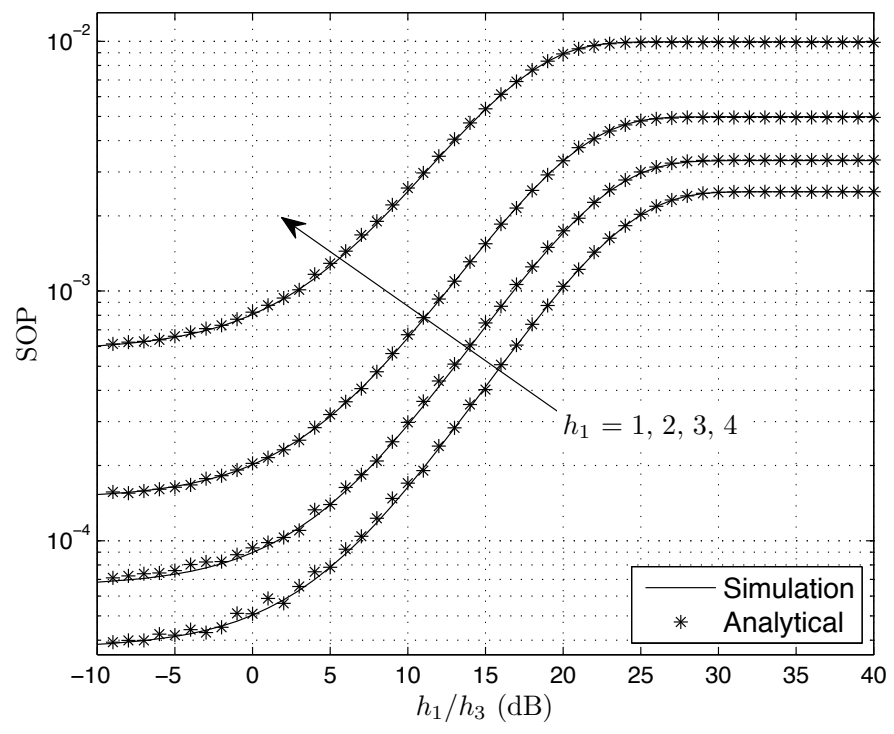

(a) $\mathrm{AF}$

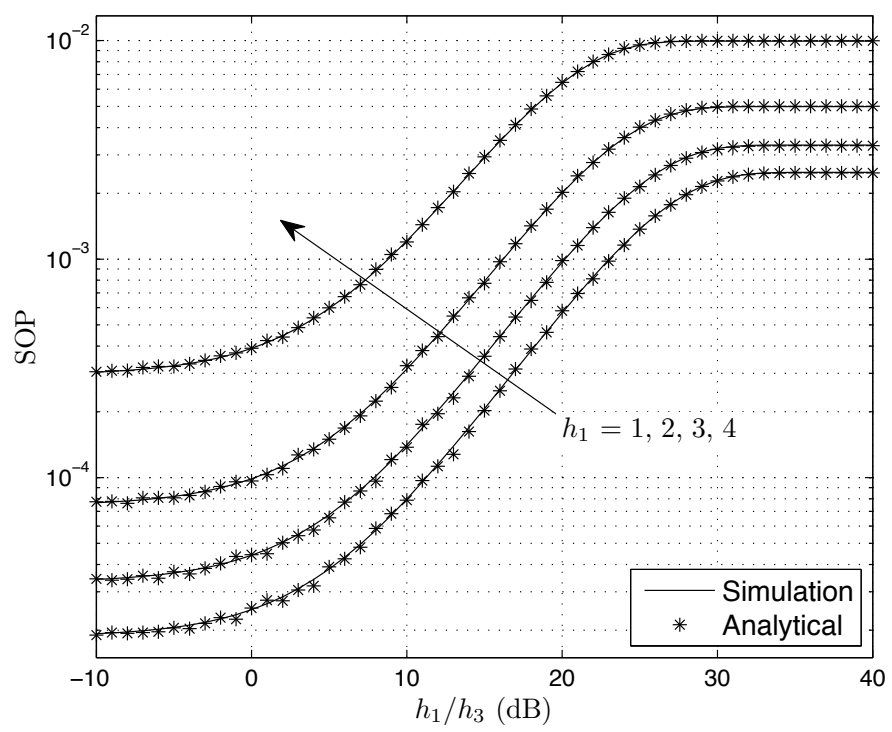

(b) $\mathrm{DF}$

Figure 4: SOP for various $h_{1}$ 
increasing $h_{1} / h_{3}$ means $R-D$ link gets worse, resulting in an increasing outage probability over $R-D$ link.

As shown in Figs. 2-4, it is clear that SOP under DF outperforms the one under AF. It is because of the decoding gain at $R$.

\section{Conclusions}

In this paper, the outage performance of a dual-hop cooperative simultaneous wireless information and power transfer (SWIPT) system was investigated under threshold AF and DF schemes. The closed-form analytical expressions for outage probability under threshold AF and DF schemes have been derived and verified through Monte-Carlo simulations.

\section{Acknowledgment}

This research was supported in part by the National Science Foundation under Grants 61401372 and 61531016, Research Fund for the Doctoral Program of Higher Education of China under Grant 20130182120017, Natural Science Foundation of CQ CSTC under Grant cstc2013jcyjA40040, the Fundamental Research Funds for the Central Universities under Grant XDJK2015B023.

\section{References}

[1] Varshney L R. Transporting information and energy simultaneously. in Proc. IEEE ISIT, 2008, pp. 1612-1616.

[2] Grover P and Sahai A. (2010). Shannon meets Tesla: Wireless information and power transfer, in Proc. IEEE ISIT, pp. 2363-2367. 
[3] Shi Q, Liu L, Xu W and Zhang R. (2014). Joint transmit beamforming and receive power splitting for MISO SWIPT systems. IEEE Trans Wireless Commu., 13(6): 3269-3280.

[4] Park J and Clerckx B. (2013). Joint wireless information and energy transfer in a two-user MIMO interference channel. IEEE Trans Wireless Commu., 12(8): 4210-4221.

[5] Zhang R, Yang L-L and Hanzo L. (2015). Energy pattern aided simultaneous wireless information and power transfer. to appear in IEEE Journal on Selected Areas in Communications, DOI 10.1109/JSAC.2015.2391551.

[6] Xing C, Wang N, Ni J, Fei Z., and Kuang J. (2013). MIMO beamforming designs with partial CSI under energy harvesting constraints. IEEE Sig Proc Lett, 20(4): 363-366.

[7] Sun Q, Li L, and Mao J. (2013). Simultaneous information and power transfer scheme for energy efficient MIMO systems. IEEE Commu Lett, 18(4): 600-603.

[8] Xiang Z and Tao M. (2012). Robust beamforming for wireless information and power transmission. IEEE Wireless Commun Lett, 1(4): 372-375.

[9] Liu C-F, Maso M, Lakshminarayana S, Lee C., T.Q.S. Quek. (2015). Simultaneous wireless information and power transfer under different CSI acquisition schemes. IEEE Trans Wireless Commu., 14(4): 19111916. 
[10] Shi Q, Xu W, Chang T-H, Wang Y, Song E. (2014). Joint beamforming and power splitting for MISO interference channel with SWIPT: An SOCP relaxation and decentralized algorithm. IEEE Trans Sig Proc, 62(12): 6194-6208.

[11] Khandaker M R A and Wong K-K. (2014). SWIPT in MISO multicasting systems. IEEE Wireless Commu Lett, 3(3): 277-280.

[12] Wang F, Peng T, Huang Y, Wang X. (2015). Robust transceiver optimization for power-splitting based downlink MISO SWIPT systems. IEEE Sig Proc Lett, 22(9): 1492-1496.

[13] Feng R, Li Q, Zhang Q, Qin J. (2015). Robust secure transmission in MISO simultaneous wireless information and power transfer system. IEEE Trans Veh Tech, 64(1): 400-405.

[14] Tian M, Huang X, Zhang Q, Qin J. (2015). Robust AN-aided secure transmission scheme in MISO channels with simultaneous wireless information and power transfer. IEEE Sig Proc Lett, 22(6): 723-727.

[15] Pan G, Tang C, Li T, and Chen Y. (2015). Secrecy performance analysis for SIMO simultaneous wireless information and power transfer systems. IEEE Trans. Commun., 63(9): 3423-3433.

[16] Khandaker M R A and Wong K-K. (2015). Robust secrecy beamforming with energy-harvesting eavesdroppers. IEEE Wireless Commu Lett, 4(1): 10-13. 
[17] Zhang R and Ho C. (2013). MIMO broadcasting for simultaneous wireless information and power transfer. IEEE Trans Wireless Commun, 12(5): 1989-2001.

[18] Gradshteyn I S and Ryzhik I M. (2007). Table of integrals, series and products, 7 Ed., San Diego: Academic Press.

[19] Abramowitz M and Stegun I A. (1970). Handbook of mathematical functions with formulas, graphs, and mathematical tables, 9th ed., New York: Dover.

[20] Prudnikov A P, Brychkov Yu A, and Marichev O I. (1992). Integrals and series, Vol. 5: Inverse Laplace transforms, New York: Gordon and Breach. 